\title{
TRANSVERSE PHASE SPACE IN THE PRESENCE OF DISPERSION
}

\author{
N. Merminga, P. L. Morton, J. T. Seeman, W. L. Spence \\ Stanford Linear Accelerator Center, \\ Stanford University, Stanford, CA 94309
}

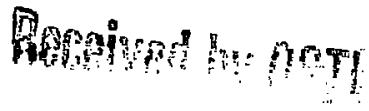

$\ln m$

\section{Abstract}

A generalized formalism is used to determine the beam ellipse parameters at some location in a transversely uncoupled transport line from a sufficient number of beam size measurements. We allow for contributions to the beam sizes from dispersion which can be either transported from some initial point, or generated along the transport line. We derive expressions for the pure betatron emittance in terms of the "effective" emittance (calculated from the measurements of the total transverse beam sizes) both in the presence and absence of bending magnetr. We give necessary and sufficient conditions for determining the pure betatron emiltance and show it to be the absolute minimum of the effective emittance. Finally we show that dispersion not only directly enlarges the emittance, but also causes a $\beta$-mismatch, resulting in further emittance dilution due to chromatic flamentation, and calculate the emittance blow-up due to the combined effect.

\section{MEASUREMENT}

Let us assume a high energy linac or transport line and let 0 denote the point where we want to determine the beam ellipse from an arbitrary number, $N$, of beam size measurements at locations $1,2, \ldots, N$ downstream of point 0 . We assume that the transfer matrix elements from point 0 to points $1,2, \ldots, N$ are known. Also we allow for contributicns to the beam sizes from dispersion which can be either transported from point 0 or generated in between.

The beam matrit $\sigma$, can be written,

$$
\sigma=\left\langle x x^{T}\right\rangle
$$

with $X$ being the vector,

$$
x=\left(\begin{array}{l}
x \\
x^{\prime} \\
6
\end{array}\right)
$$

where we have restricted ourselves to the $x$-plane and energy degrees of freedom. The average is over the beam

'Work supporled by' the Department of Energy, contract DE-AC03-76SF00515. phase space. The beam matrix propagates from point 0 to point $i$ according to the equation:

$$
\sigma^{i}=R^{i} \sigma^{0}\left(R^{i}\right)^{T} \quad i=1,2, \ldots, N
$$

where $R^{i}$ is the transfer matrix from 0 to $i$. We also define the transfer matrix from $i$ to $j$ as $R^{i j}$ for later use. For a transversely uncoupled transport line and in the absence of acceleration, the relevant part of the above equation is explicitly, in TRANSPORT [1] notation.

$$
\begin{aligned}
& \left(\begin{array}{lll}
\sigma_{11} & \sigma_{12} & \sigma_{16} \\
\sigma_{12} & \sigma_{22} & \sigma_{26} \\
\sigma_{16} & \sigma_{26} & \sigma_{66}
\end{array}\right)=\left(\begin{array}{ccc}
R_{11} & R_{12} & R_{16} \\
R_{21} & R_{22} & R_{26} \\
0 & 0 & 1
\end{array}\right) \\
& \times\left(\begin{array}{lll}
\sigma_{11} & \sigma_{12} & \sigma_{16} \\
\sigma_{12} & \sigma_{22} & \sigma_{26} \\
\sigma_{16} & \sigma_{26} & \sigma_{66}
\end{array}\right)_{(\mathrm{D})}\left(\begin{array}{lll}
R_{11} & R_{21} & 0 \\
R_{12} & R_{22} & 0 \\
R_{16} & R_{26} & 1
\end{array}\right) .
\end{aligned}
$$

In particular the square of the width of the beam is:

$$
\begin{aligned}
\sigma_{11}^{i}= & \left(R_{11}^{i}\right)^{2} \sigma_{11}^{0}+\left(R_{12}^{i}\right)^{2} \sigma_{22}^{0}+2 R_{11}^{i} R_{12}^{i} \sigma_{12}^{0}+ \\
& 2 R_{11}^{i} R_{16}^{i} \sigma_{16}^{0}+2 R_{12}^{i} R_{16}^{i} \sigma_{26}^{0}+\left(R_{15}^{i}\right)^{2} \sigma_{66}^{0}
\end{aligned}
$$

or in matrix notation

$$
\sigma_{11}^{i}=L_{a}^{i} \sigma_{a}^{0},
$$

where $\sigma_{a}^{0}$ is the 6 -component vector

$$
\sigma_{a}^{0}=\left(\sigma_{11}^{0}, \sigma_{22}^{0}, \sigma_{12}^{0}, \sigma_{16}^{0}, \sigma_{26}^{0}, \sigma_{66}^{0}\right) \text {. }
$$

If the elements of the trans[er matrix vary sufficiently from point to point, then for $N=6, L$ is invertible and one can determine the beam ellipse at point 0 ,

$$
\sigma_{a}^{0}=\left(L^{-1}\right)_{a}^{i} \sigma_{11}^{i} \text {. }
$$

(If $N>6$ the parameters are overdetermined but they can be solved for in a least squares fit.)

The elements of the beam matrix can be generally parametrized in terms of the Tuiss parameters $\beta_{0} . a_{0} .70$ and the dispersion function $\eta_{0}$.

$$
\begin{aligned}
& \sigma_{11}^{0}=\epsilon_{\beta} \beta_{0}+\eta_{0}^{2}\left\langle\delta^{2}\right\rangle \\
& \sigma_{22}^{0}=\epsilon_{\beta} \gamma_{0}+\eta_{0}^{\prime 2}\left\langle\delta^{2}\right\rangle \\
& \sigma_{12}^{0}=-\epsilon \beta \alpha_{0}+\eta_{0} \eta_{0}^{\prime}\left\langle\delta^{2}\right\rangle \\
& \sigma_{16}^{0}=\eta_{0}\left\langle\delta^{2}\right\rangle \\
& \sigma_{26}^{0}=\eta_{0}^{\prime}\left(\delta^{2}\right\rangle \\
& \sigma_{66}^{0}=\left\langle\delta^{2}\right\rangle,
\end{aligned}
$$

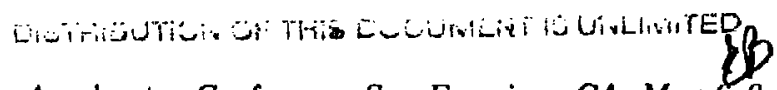

Presented at the IEEE Particle Accelerator Conference, San Francisco, CA. May 6-9. J951 
where $\alpha_{0}=-1 / 2 \beta_{0}^{\prime}$ and $\beta_{0} \gamma_{0}=1+\alpha_{0}$. The "effective" transverse emittance in terms of the total beam matrix elements is

$$
\tilde{\epsilon}_{0}^{2}=\sigma_{11}^{0} \sigma_{22}^{0}-\left(\sigma_{12}^{0}\right)^{2} .
$$

which is given in terms of the pure betatron emittance by [2]

$$
\dot{\epsilon}_{0}^{2}=\epsilon_{\beta}^{2}+\epsilon_{0} \frac{\eta_{0}^{2}+\left(\beta_{0} \eta_{0}^{\prime}+\alpha_{0} \eta_{0}\right)^{2}}{\beta_{0}}\left\langle\delta^{2}\right\rangle .
$$

Since $\eta_{0}, \eta_{0}^{\prime}$ and $\left(\delta^{2}\right)$ can be determinea from eq. (8), one can separate out the pure transverse betatron emittance co. $_{0}$ It is evident that this separation is made possible by the presence of bends, i. e. a known, non-trivial $R_{10}$. On the other hand if $R_{16}^{i_{6}}=0$, the above equations reduce to the usual 3-eomponent formalism [3] in which only $\tilde{c}_{0}$ (and not $c_{p}$ ) can be determined.

The general conditions on $R_{16}$ to achieve the separation of the pure betatron contribution to the emittance will now be specified. If 0 is a reference point of the transport line, let $b$ be a downstream point, upstream of which there is at least one bend of known strength, and downstream of which there are no bends at least as far as measurement location $i$. Then

$$
R_{16}^{1}=R_{11}^{i b} R_{16}^{b}+R_{12}^{i b} R_{26}^{b} .
$$

and the $R_{16}$ dependent terms in eq. (5) become,

$$
\begin{aligned}
2 R_{16}^{i} & \left(R_{11}^{i} \sigma_{16}^{0}+R_{12}^{i} \sigma_{26}^{0}\right)+\left(R_{16}^{i}\right)^{2} \sigma_{66}^{0}= \\
& 2\left(R_{11}^{i b} R_{16}^{b}+R_{12}^{b} R_{26}^{b}\right) \\
& \times\left[R_{11}^{i b}\left(R_{11}^{b} \eta_{0}+R_{12}^{b} \eta_{0}^{\prime}+\frac{1}{2} R_{16}^{b}\right)\right. \\
& \left.+R_{12}^{i b}\left(R_{21}^{b} \eta_{0}+R_{22}^{b} \eta_{0}^{\prime}+\frac{1}{2} R_{26}^{b}\right)\right]\left(\delta^{\eta}\right) .
\end{aligned}
$$

The two independent combinations of the three unlnowns in the above expression,

$$
\left(R_{k 1}^{b} \eta_{0}+R_{k 2}^{b} \eta_{0}^{\prime}+\frac{J}{2} R_{k 6}^{b}\right)\left(\delta^{2}\right\rangle \quad \text { for } \quad k=1,2 ，
$$

can be determined given two measurements separated by sufficient phase advance. The presence of an additional bending magnet downstream of $i$, followed by at least one additional measurement is then necessary and sufficient to separately determine $\left(\delta^{2}\right)$. Thus at least two bending magnets and three measurements downstream of the first, with at least one measurement between the two bends, are required to fully determine the pure betatron emittance. This condition applies equally to the situation where quadrupole atrengths are varied to provide phase shifts that allow the sampling of different phases in the beam from observations in fewer locations. However one could use only one bending magnet if quadrupoles both upstream and downstream of it are varied simultaneously [4], or if the bending magnet itself is varied.

\section{EMITTANCE}

It is interesting to calculate the effective emittanr: throughout the region in which the measurements are taken,

$$
\bar{\epsilon}_{i}^{2}=\sigma_{11}^{i} \sigma_{22}^{i}-\left(\sigma_{12}^{i}\right)^{2} \text {. }
$$

in terms of the total dispersion function which includecontributions from propagation of $\eta_{0}$ and $\eta_{0}^{\prime}$. and any bends present in the beam line; namely

$$
\begin{aligned}
& \eta_{i}=R_{11} \eta_{0}+R_{12} \eta_{0}^{\prime}+R_{16} . \\
& \eta_{i}^{\prime}=R_{21} \eta_{0}+R_{22} \eta_{0}^{\prime}+R_{26} .
\end{aligned}
$$

The effective emittance at point $i$ is then given by the same form as eq. (11)

$$
\frac{\bar{\epsilon}_{i}^{2}}{c_{\beta}^{2}}=1+\frac{\eta_{i}^{2}+\left(\beta_{1} \eta_{i}^{\prime}+\alpha_{i} \eta_{2}\right)^{2}}{c_{\beta} \beta_{i}}\left\langle\delta^{2}\right\rangle .
$$

which can be different for different locations in the transport line.

Suppose now that there are no bends between point 0 and point $i$, therefore dispersion is not generated between 0 and $i$. However $\eta_{0}$ and $\eta_{0}^{\prime}$ can propagate downstream from point 0 . Going back to eqs (15), $\eta_{1}$ and $\eta_{1}^{\prime}$ at point $i$ are now given by the relations

$$
\begin{aligned}
& \eta_{h}=R_{11} \eta_{0}+R_{12} \eta_{0}^{\prime} . \\
& \eta_{i}^{\prime}=R_{21} \eta_{0}+R_{22} \eta_{0}^{\prime} .
\end{aligned}
$$

Also transforming the Twiss parameters back to point 0 [5] we get,

$$
\bar{\epsilon}_{i}=\bar{\epsilon}_{0},
$$

i. e. $I$ is independent of position along the bean line Although $\eta_{i}$ and $\eta_{i}^{\prime}$ are now different from those in eqs (15). the combination $\left[\eta_{i}^{2}+\left(\beta_{i} \eta_{i}^{\prime}+\alpha_{i} \eta_{i}\right)^{2}\right] / \beta_{i}$ in eq. (16) remain. invariant (an example of the Courant-Snyder invariant). With the use of orthogonal dispersion controls, each of the two terms in the sum. $\eta$ and $\left(\not \vec{\beta} \eta^{\prime}+a \eta\right)$, can be separately zeroed and so one arrives at the absolute minimum of the effective emiltance, $c \beta$. The solid line of the top plots of fig. 1 show the variation of the effective emittance $\bar{c}_{s} w$ ith $\eta_{0}$ and $\eta_{0}^{\prime}$. SLAC linac parameters have been used as an example.

\section{FILAMENTATION}

In addition to the effective emittance $\bar{t}$ there is an 7-dependeut effective $\beta$-function $\dot{B}$, such that

$$
\begin{aligned}
& \sigma_{11}=\overline{\bar{c}} \bar{\beta} . \\
& \sigma_{12}=-\bar{c} \bar{a} .
\end{aligned}
$$

which is shown pictoriaily in fig. 2. The phase space is thumismatched with respect to that described by $\beta$. against which $\bar{\beta}$ may be said to 'beat.' The invariant fractional $\beta$-beat amplitude $\|\Delta \beta / \beta\|$, in terms of.which

$$
\bar{\beta} / \beta=\|\Delta \beta / \beta\| \cos \left(2 \Delta \psi+\psi_{0}\right)+\left(1+\|\Delta \beta / \beta\|^{2}\right)^{1 / 2}
$$


and

$$
\bar{\alpha}-(\bar{\beta} / \beta) \alpha=\|\Delta \beta / \beta\| \sin \left(2 \Delta \psi+\psi_{0}\right) .
$$

where $\Delta \psi$ is the betatron phese advance, is then closely related to the emittance increase

$$
\begin{aligned}
\left(1+\left\|\frac{\Delta \beta}{\beta}\right\|^{2}\right)^{1 / 2} & =\frac{1}{2}\left[\frac{\bar{\beta}}{\beta}+\frac{\beta}{\bar{\beta}}\left(1+\left(\overline{\mathrm{a}}-\frac{\bar{\beta}}{\bar{\beta}} \alpha\right)^{2}\right)\right] \\
& =\frac{1}{2}\left(\frac{\bar{\epsilon}}{\bar{c}}+\frac{\epsilon \bar{\beta}}{\tilde{\epsilon}}\right),
\end{aligned}
$$

where $z / c_{\rho}$ is given by eq. (11). Its dependence on $\eta_{0}$ and $\eta_{0}^{\prime}$ is shown in the bottom plots in fig. 1 . For small values it is seen to be of second order in $\left[\eta^{2}+\left(\beta \eta^{\prime}+a \eta\right)^{2}\right] / \beta \epsilon_{\beta}$.

If the beam with incoming effective emittance $\bar{\varepsilon}_{0}$ and the associated $\beta$-miamalch travels through a periodic focusing lattice, the phase space filaments due to chromatic phase decoherence and a new effective emitlance $\overline{\bar{\epsilon}}$, given by [6]

$$
\frac{\overline{\bar{c}}}{\overline{\bar{\epsilon}_{0}}}=\left(1+\left\|\frac{\Delta \beta}{\beta}\right\|^{2}\right)^{1 / 2}
$$

is reached. Using eq. (22) the total emittance growth is (to all orders)

$$
\frac{\tilde{\epsilon}}{c_{\rho}}=1+\frac{1}{2} \frac{\eta_{0}^{2}+\left(\beta_{0} \eta_{0}^{\prime}+\alpha_{0} \eta_{0}\right)^{2}}{c_{\rho} \beta_{0}}\left(\varepsilon^{2}\right) .
$$

The dashed lines of the top plots of fig. 1 show the variation of $\bar{c}$ as a function of $\eta_{0}$ and $\eta_{0}^{\prime}$. Since the $\beta$-mismatch is of second order, the lowest order total emittance growth is still given by eq. (11).

\section{Acknowledgements}

Some aspects of this work have been considered earlier by one of the authors (P. M.) and Lenny Rivtin. We would like to thank Martin Lee and Ron Ruth for illuminaling discussions on this issue and for reading and commenting on the manuscript.

\section{REFERENCES}

[1] K. L. Brown et al, TRANSPORT, A Computer Pro gram for Designing Charged Pa:ticle Beam Transport Systems, SLAC-91, May 1977.

[2] J. C. Sheppard, $\eta$ in the Linac, SLAC Internal Note CN-298, April 1985.

[3] J. Seeman, Determination of $\epsilon, \beta, \alpha$ from an Arbitrary Number of Beam Size Measurements, SLAC Internal Note CN-376, April 1990.

[4] J. T. Seeman and C. Adolphsen, Beam Phase Space Determination at $50 \mathrm{GeV}$ in the SLC Linac, SLAC-PUB4642, May 1988.

[5] K. L. Brown and R. V. Servrancke, First-and Secondorder charged Particle Opties, SLAC-PUB-3381, July 1984.

[6] W: L. Spence, unpublished.

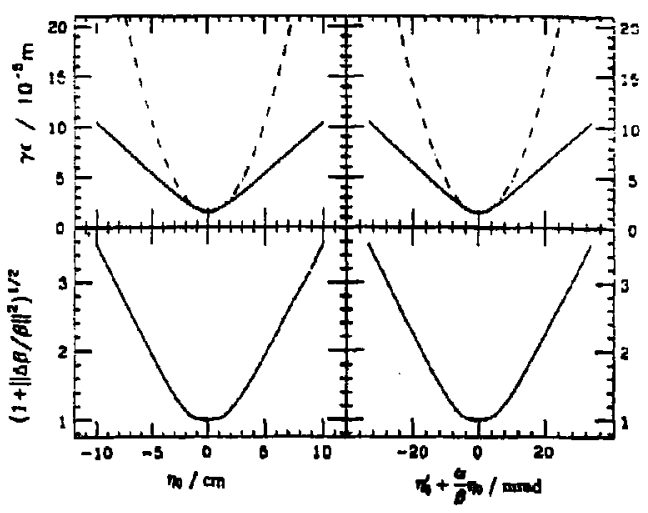

Figure 1: (Top) The SLAC Linear Collider (SLC) effective notmalized emittance immediacely after re-injection into the linac (aolid), and after sufficient phase advance for chromatic filanentation of the ascocialed $\beta$-mismatch to have taken place (dasbed); $\beta \simeq 3 \mathrm{~m}, \alpha \simeq 0, c_{\beta} \simeq 7000$ $\mu \mathrm{m}-\mu \mathrm{rad}$, and $E / m c^{2}=2300$. (Bottom) The $\beta$-mismatch factor for the same parameter values.

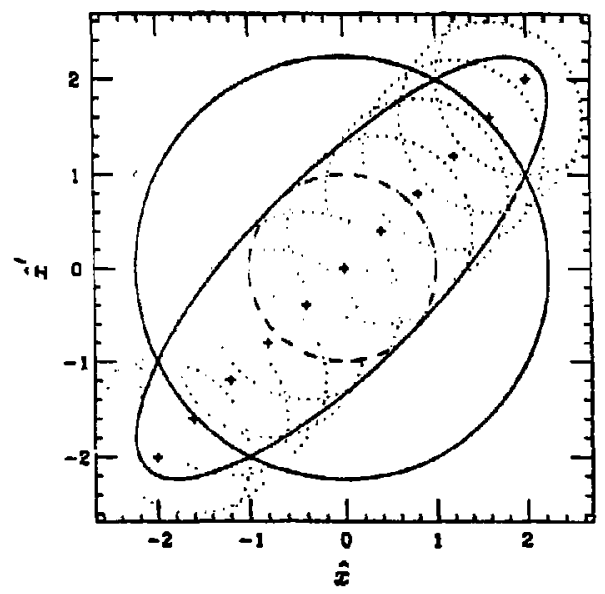

Figure 2: The betatron phase ellipse (dashes) in normal coordinates $\dot{x}=x / \sqrt{\beta}, \dot{x}^{\prime}=\left(\beta x^{\prime}+\alpha x\right) / \sqrt{\beta}$ is effectively increased in ares and distorted by dispersion. The series of dotted circles correspond to different energies $|\delta| \leq\left(\delta^{2}\right\rangle^{1 / 2}$ and the solid ellipse represents the effective phase space in eq. (19). The large circle, with a radius equal to the average of the major and minor semi-axes of the solid ellipse. then corresponds to the equivalent filamented phase space given by eq. (24). 